SLAC-PUB-10825

October 2004

\title{
THE GLAST GAMMA-RAY OBSERVATORY
}

\author{
L. LATRONICO \\ on behalf of the GLAST Collaboration \\ INFN Pisa and University of Pisa, \\ Via Buonarroti 2, \\ 56100 Pisa, Italy \\ E-mail: luca.latronico@pi.infn.it
}

\begin{abstract}
GLAST is a space mission that will observe the gamma-ray sky between $20 \mathrm{MeV}$ and $1 \mathrm{TeV}$ with unprecedented resolution and sensitivity. The Large Area Telescope (LAT), the main instrument onboard the GLAST satellite, is built with stateof-the-art particle physics detectors, and combines a large area si-strip trackerconverter, that will measure direction of incoming photons to an imaging CsI e.m. calorimeter for measurements of photon energies; an outer, segmented AntiCoincidence Detector will reject charged particle background. In this paper we give an overview of the many physics goals and potential reach of the GLAST observatory and describe in detail the instrument design and performance.
\end{abstract}

\section{Motivation for the GLAST mission}

In the past decades, observation of the sky at progressively higher photon energies revealed unexpectedly rich phenomenologies. It was only after the beginning of satellite experiments that it became possible to detect high energy gamma-rays, in the $\mathrm{MeV}-\mathrm{GeV}$ range, before they converted to lower energy, secondary particles after interacting with the earth atmosphere. Few pioneering satellite missions, like COS-B, had detected a diffused $\gamma$ ray emission from the galaxy, up to $\mathrm{GeV}$ energies, but it was EGRET, the first dedicated detector, that was able to partially resolve this emission into few hundreds point sources of various classes, located at galactic as well as extragalactic coordinates $\left({ }^{1}\right)$. The sources revealed by EGRET exhibit a wide variety of characteristics, both in their energy spectra and their time variability, ranging from steady-state black-holes and blazars to supernovae, pulsars or even very short, finely structured transient emissions like gammaray bursts (GRB) or flares from Active Galactic Nuclei (AGN). Moreover most of them remain unidentified, as a direct association to known sources or classes of sources at other wavelengths was not possible, due to the

Work supported in part by the Department of Energy Contract DE-AC02-76SF00515

Stanford Linear Accelerator Center, Stanford University, Stanford, CA 94309 
limited angular and energy resolution of the detectors onboard EGRET.

The GLAST mission $\left({ }^{2}\right)$ is a gamma-ray telescope that will have optimized performance for detection and measurement of photons in the $20 \mathrm{MeV}-1 \mathrm{TeV}$ energy range, due to a specific design which makes extensive use of high-resolution detectors from particle physics instrumentation. The Large Area Telescope ( $L A T$ ), the main instrument onboard the GLAST satellite, will allow precise source localization and spectral analysis, as well as prompt transients detection and measurement. The overall improvement in sensitivity with respect to previously flown missions will be of more than an order of magnitude, thus offering a powerful probe into the engine of nature's highest energy processes.

\section{Science reach}

A full-sky survey with the LAT is planned for the first year of the GLAST mission. The excellent angular resolution and sensitivity of the LAT telescope will provide high-quality data with large statistics for detailed sky mapping, with source localization to the level of arc-minutes, thus enabling GLAST to solve the problem of the unidentified sources. Most of the 271 $\gamma$-ray sources in the third EGRET catalog are in fact still unidentified; the large error boxes in the localization do not allow unambiguous association of these sources to known objects in the optical, radio, or X-ray bands. Moreover the distribution of the sources on the sky and their variable temporal properties suggest that more than one source class is required. While there are indications that many of these sources belong to well established classes like blazars, radio-quiet pulsars, or binary systems, some of them may very well be examples of previously undetected $\gamma$-ray emitters, like forming clusters of galaxies or the recently discovered Galactic microquasars.

A large fraction of the known high-energy sources is instead represented by AGNs, most of them belonging to the blazar class and characterized by jet-like emission and broadband spectra peaking at gamma rays and between radio and X-ray. The high sensitivity and large effective area of the LAT will be crucial for testing different models proposed for the central engine of blazars. Detailed spectral studies of gammas emitted should allow distinction between pure leptonic and hadronic particle acceleration mechanism.

Extrapolation of the $\log N$-log $S$ relation $\left({ }^{3}\right)$ for AGNs suggests that thousands of new AGNs will be detected by the LAT. With such a large population, the redshift dependence of the $\gamma-\gamma$ attenuation of AGN spec- 
tra by the infrared-UV Extragalactic Background Light may be useful for measuring this cosmic background.

It is widely projected that GLAST will discover $\simeq 250$ new pulsars, and should allow direct pulsation searches in the $\gamma$-ray band, thanks to its high time resolution and detection efficiency. The large effective area of the LAT will also provide high statistics spectra, opening the way to a clear discrimination between the outer gap and polar cap models of $\gamma$-ray production for rotation-powered pulsars.

After resolving the gamma-ray sky into point sources, GLAST will then allow study of the residual gamma-ray background, whose existence, if confirmed, might bring to discovery of exotic particles relic densities or annihilation of dark matter WIMPS $\left({ }^{4}\right)$.

The accurate time resolution of GLAST will allow study of transient phenomena, like solar flares or Gamma-ray bursts (GRBs). In recent years, GRBs have been shown to be immensely energetic $\left(10^{54} \mathrm{erg}\right)$ outbursts that occur at cosmological distances. Bursts have highly variable temporal structures $(\sim \mathrm{ms})$ and typical durations of seconds. The large effective area and field of view of the LAT, together with the unprecedently short instrumental deadtime, will greatly advance knowledge of GRBs at high energies. The highest-energy emissions constrain the acceleration processes and the bulk motions within the fireballs. LAT data will permit distinguishing between internal and external shock models for the production of gamma rays, and evaluation of the baryon fraction in the fireballs. The GLAST observatory will be equipped with a second instrument, the Gamma-Burst Monitor $(G B M)$, specifically developed for gamma-ray bursts studies. The GBM, based on a virtually $4 \pi$, dead-time-less array of NaI and BGO scintillators, will cover an energy range between $10 \mathrm{KeV}$ and $100 \mathrm{MeV}$, thus completing observations from the LAT and providing real-time alerts.

The combined resolution and efficiency for spectral studies and the high localization power will be crucial for studying the origin and acceleration sites of hadronic cosmic rays. Cosmic rays (CR) have been known and measured for a long time. A fundamental outstanding question is the origin of the hadronic CRs, which is widely believed, but not yet proven to be supernova remnants (SNR). The SNR origin for nucleons would have a signature in a broad peak at $68 \mathrm{MeV}$ from by the decay of $\pi^{0}$ produced by the interaction of freshly-accelerated CR nucleons with interstellar gas in the vicinity of an SNR. The LAT should be able to spatially resolve several SNR with degree-scale angular extents and spectrally resolve the $\pi^{0}$ decay peak from other $\gamma$-ray components. 


\section{The Large Area Telescope}

The LAT has to efficiently measure direction and energy of gammas from the sky in a wide energy range, and for this purpose the pair conversion telescope design was adopted. It is based on a high resolution tracker, composed by a sequence of thin, high-Z material converters closely followed by $\mathrm{X}-\mathrm{Y}$ detection planes, coupled to an imaging electromagnetic calorimeter (CAL): when high energy photons $(>10 \mathrm{MeV})$ cross the telescope they interact with one of the converters producing $e^{+} e^{-}$pairs, and their direction is reconstructed by measuring the leptons tracks and energies. The telescope is surrounded by an anticoincidence detector (ACD), used to reject the background of charged particles, about $10^{5}$ times greater than the expected $\gamma$-ray flux. Key to a good background rejection is a very high MIP detection efficiency, which is constrained to have an average value $>0.9997$ for the whole ACD surface $\left(>8 \mathrm{~m}^{2}\right)$.

The GLAST LAT has a highly modular structure, being composed by an array of $4 x 4$ identical towers, each with an area of $37 x 37 \mathrm{~cm}^{2}$ and equipped with a tracker, a calorimeter and a custom read-out electronics module. Calorimeter towers are built with CsI logs, organized in an hodoscopic configuration to ensure efficient 3D shower reconstruction over several orders of magnitude in energy. The ACD around the telescope, which is built with plastic scintillators tiles, is segmented so that a signal from a specific tile can be correlated to the track direction; this avoids the self-veto effect observed with monolithic ACDs, like that of EGRET, which severely limited the sensitivity at high energies due to the backsplash of particles from the calorimeter. The LAT towers are self-triggered when a signal is detected in any three consecutive $\mathrm{X}-\mathrm{Y}$ tracker planes; further trigger selection are applied using events from all 16 towers and the ACD data, in order to reduce the raw trigger rate from few $\mathrm{KHz}$ to few $\mathrm{Hz}$.

\subsection{The LAT Tracker}

The LAT tracker is the largest ever built for space applications, with an overall area close to $80 \mathrm{~m}^{2}$ and a total number of channels approaching $10^{6}$. Modularity is therefore extended to all possible levels in the LAT towers, in order to maximize production reliability and simplify the assembly, while keeping costs at a reasonable level. Each tracker tower is composed by a sequence of $18 \mathrm{X}-\mathrm{Y}$ detection planes, obtained by stacking 19 composite panels (trays), each equipped with a tungsten (W) converter and Si-strip detectors on both sides, oriented in the same direction. During tower as- 
sembly, trays are mounted at $90^{\circ}$ with respect to one another, so that each $\mathrm{W}$ converter is followed by an $\mathrm{X}$ and a Y Si-layer, only spaced by a $2 \mathrm{~mm}$ gap. The $\mathrm{W}$ thickness vary across the tower, with, top to bottom, 12 planes with a $3 \%$ R.L., 4 with $18 \%$ R.L. and 2 with no converter. The tracking layers are obtained with 4 baseline Si-strip detectors (ladders), mounted side by side and connected to the front-end electronics located on the tray side. Each ladder is obtained by wire-bonding four Si-strip sensors (SSD) in a line, as to have $35.8 \mathrm{~cm}$ long strips (fig. 1).

The design of the LAT tracker rely on large area, single-sided Si-strip detectors, which can nowadays be produced with a very high quality at acceptable costs. Use of Si-strip sensors has other advantages which greatly impact the LAT performance. They have an intrinsic high resolution and don't make any use of consumables. The signal can be read out with fast-shaping electronics, so that the LAT instrumental dead time can be reduced to $<100 \mu s$, well suited for detection of transients on the ms scale, typical of GRBs and flares. The modest level of background radiation in space does not introduce any significant deterioration of the signal with time. Furthermore, very thin detecting elements such as silicon layers give a favourable aspect ratio to the telescope, yet allowing a stack of many high resolution sensitive planes, so that photons from a wide field of view cross enough layers for efficient reconstruction. This much increases the statistics of detected photons, with benefits on the sensitivity and the resolution of the LAT, ultimately increasing the science potential reach.

The strip pitch was chosen to be $228 \mu m$, a good compromise between the requirement of a high resolution, the unavoidable limitation on resolution due to the multiple scattering introduced by the $\mathrm{W}$-converters and the need to limit to overall number of channels. From a 6 inches, high resistivity $(<5 K \Omega / \mathrm{cm})$ Si-wafer, $384 p^{+}$strips are implanted on a n-type substrate, biased through polysilicon resistors and AC-coupled to the frontend electronics.

Hamamatsu Photonics has produced all the SSDs $(\simeq 11500)$ for the LAT Tracker, with excellent quality and production yield. $80 \%$ of the sensors produced so far were tested by measuring their leakage current, bulk capacitance and depletion voltage as a function of the applied bias, and by measuring the strips pattern alignment with the wafer cut: the overall number of rejected wafers after all qualification test was $\simeq 0.6 \%\left({ }^{4}\right)$.

The following production step, e.g. assembly of the tracker Si-strip ladders, require high quality standards for the construction of more than 2700 detectors, each of them involving many delicate operations like han- 


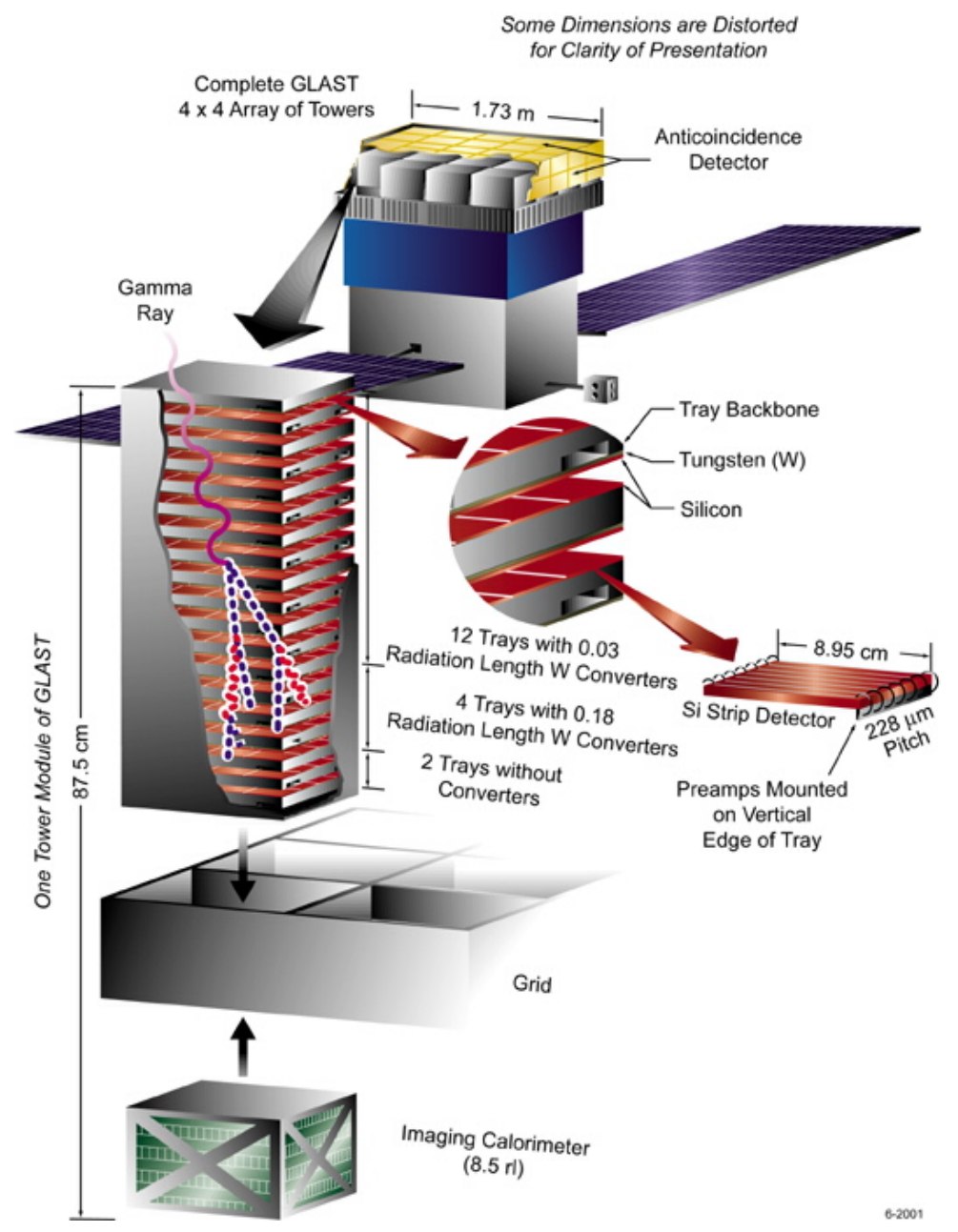

Figure 1. The GLAST-LAT detector

dling, alignment and gluing of the silicon sensors, microbonding of $>1500$ Si-strips, bonding encapsulation. It was therefore decided to develop assembly procedures in collaboration with space-qualified companies which would eventually build the ladders, leaving the quality control to the institutes collaborating in the project. Two italian companies were selected, and the production of the first 500 ladders gave excellent results, matching very well with what expected from the composing SSDs. Encapsulation of bonds did not change the detectors properties, and only produced a slight 
increase in the current for some ladders, as expected.

The design of mechanical trays is now well established, and makes use of carbon fiber for the top and bottom panels, carbon-carbon for the structure close-outs and $\mathrm{Al}$ for the honeycomb core; this solution offers high resistance to mechanical stresses, high thermal conductivity for heat dissipation and low material budget. Prototypes produced with this design had excellent planarity $(<20 \mu m)$ and successfully passed specific space qualification environmental tests, e.g. thermal $\left(-30^{\circ} \mathrm{C},+50^{\circ} \mathrm{C}\right)$ and vibrational $(0.02,2 \mathrm{KHz})$ cycles $\left({ }^{4}\right)$. The first flight trays were equipped with dummy silicon sensors and have been assembled into a mechanical tracker tower, which outperformed alignemnt specifications and will soon be thouroughly environmentally tested.

A live minitracker tower with six silicon layers was instead built with flight silicon sensors and read-out electronics. This is built with lowpower $(<240 \mu \mathrm{W} / \mathrm{ch})$, custom design ASICs, and has on-board digital zerosuppression to cope with the limited bandwidth available on the satellite for downloading data to ground.

The minitracker was succesfully integrated with a calorimeter prototype and exposed to cosmic rays and $17.6 \mathrm{MeV} \gamma$, showing excellent performance for detection efficiency and noise occupancy.

\section{Conclusions}

The Large Area Telescope of the GLAST mission is a very good example of a successfull partnership between the HEP and Astrophysics communities. A careful design and use of state-of-the-art particle detectors will give the LAT superior angular and energy resolution, enabling a high resolution exploration of the rich yet still poorly explored gamma-ray sky. Particularly important for this achievements will be the LAT silicon tracker, whose construction is on schedule for the 2006 launch, with results far better than the project specifications.

\section{References}

1. P. Nolan et al., IEEE Trans. Nucl. Sci., 39, 993-996

2. E. Bloom et al., Proposal for the Gamma-ray Large Area Space Telescope, SLAC-R-22, Feb. 1998 - see also http://glastserver.pi.infn.it/glast

3. Stecker F.W., Salamon M.H. 1996, ApJ 464, 600

4. A. Morselli, F. Gargano, S. Raino', these proceedings 